\title{
Perfil sérico hormonal de un dispositivo intravaginal liberador de progesterona en ovejas
}

\author{
Gómez, M.V.'; Soto, A.T.'; Migliorisi, A.L.'; Mestorino, N. ${ }^{3}$; Sota, R.L. ${ }^{1,2}$ \\ ${ }^{1}$ Instit. Investig. Reprod. Anim. (INIRA), Fac. Cs. Vet. Univ. Nac. \\ La Plata, Argentina. ${ }^{2}$ Laborat. Estud. Farm. y Toxicol. FCV-UNLP. ${ }^{3}$ CONICET, Buenos Aires, Argentina. \\ E-mail: verano@fcv.unlp.edu.ar
}

\begin{abstract}
Resumen
Gómez, M.V.; Soto, A.T.; Migliorisi, A.L.; Mestorino, N.; Sota, R.L. Perfil sérico hormonal de un dispositivo intravaginal liberador de progesterona en ovejas. Rev. Vet. 32: 2, 206-210, 2021. Los dispositivos intravaginales (DIV) con $300 \mathrm{mg}$ de progesterona $\left(\mathrm{P}_{4}\right)$ pueden reutilizarse en los protocolos de sincronización en ovinos con una duración de hasta 7 días. La reutilización de DIV con menor concentración hormonal, así como estudios farmaco-cinéticos a través del análisis no compartimental de las concentraciones hormonales luego de la colocación de dispositivos de $1^{\circ} \mathrm{o} 2^{\circ}$ uso no han sido descriptos previamente. El objetivo del presente trabajo fue evaluar la utilización y reutilización de dispositivos intravaginales con una concentración inicial de $160 \mathrm{mg}$ de $\mathrm{P}_{4}$. Para ello se utilizaron 20 ovejas que conformaron cinco grupos: control (sin DIV), CIDN (DIV $300 \mathrm{mg} \mathrm{P}_{4} 1^{\circ}$ uso), CIDU (DIV $300 \mathrm{mg} \mathrm{P}_{4} 2^{\circ}$ uso), CRON (DIV $160 \mathrm{mg} \mathrm{P}_{4} 1^{\circ}$ uso) y CROU (DIV $300 \mathrm{mg} \mathrm{P}_{4} 2^{\circ}$ uso). Las valoraciones séricas hormonales se realizaron a través de radioinmunoensayo. La concentración máxima y el tiempo medio de residencia fueron similares entre CIDN Y CRON, en cambio CIDU presentó una concentración máxima y un tiempo medio de residencia mayor que CROU. Además, CROU presentó concentraciones séricas sub-luteales a partir de las 48 h de colocado y liberó un $68,5 \%$ menos de $\mathrm{P}_{4}$ que CIDU y un $72,5 \%$ menos que CRON. En base a los resultados obtenidos, la reutilización de DIV con menor concentración hormonal provocaría niveles sub-luteales que posiblemente afectarían la fertilidad.
\end{abstract}

Palabras clave: dispositivo intra-vaginal, reuso, progesterona, ovinos.

\begin{abstract}
Gómez, M.V.; Soto, A.T.; Migliorisi, A.L.; Mestorino, N.; Sota, R.L. Evaluation of the serum hormonal profile with a progesterone-releasing intravaginal device in sheep. Rev. Vet. 32: 2, 206-210, 2021. Intravaginal devices (IVD) with $300 \mathrm{mg}$ of progesterone $\left(\mathrm{P}_{4}\right)$ can be reused in sheep synchronization protocols with a duration of up to 7 days. The reuse of IVD with lower hormonal concentration, as well as pharmaco-kinetic studies through non-compartmental analysis of hormonal concentrations after the insertion of 1st or 2nd use devices have not been described previously. The objective of this work was to evaluate the use and reuse of IVD with an initial concentration of $160 \mathrm{mg}$ of $\mathrm{P}_{4}$. For this, 20 sheep were used that made up five groups: control (without DIV), CIDN (IVD $300 \mathrm{mg} \mathrm{P} 1_{4} 1^{\text {st }}$ use), CIDU (IVD 300 $\mathrm{mg} \mathrm{P}_{4} 2^{\text {nd }}$ use), CRON (IVD $160 \mathrm{mg} \mathrm{P}_{4}{ }^{\text {st }}$ use), and CROU (IVD $300 \mathrm{mg} \mathrm{P}_{4} 2^{\text {nd }}$ use). Hormonal serum samples were analyzed by radioimmunoassay. The maximum concentration and the mean residence time were similar between CIDN and CRON, whereas CIDU presented a maximum concentration and a mean residence time greater than CROU. Also, CROU presented sub luteal serum concentrations $48 \mathrm{~h}$ after placement and released $68.5 \%$ less $\mathrm{P} 4$ than CIDU and $72.5 \%$ less than CRON. Based on the results obtained, the reuse of IVD with a lower hormonal concentration would cause sub luteal levels that could affect fertility.
\end{abstract}

Key words: intra-vaginal device, reuse, progesterone, sheep

\section{INTRODUCCIÓN}

La utilización de dispositivos intravaginales (DIV) de liberación de progesterona $\left(\mathrm{P}_{4}\right)$ para la sincronización de celos en ovinos, comenzó a mediados de la década de 1980 como una alternativa a las esponjas intravaginales ${ }^{7}$. Estudios realizados en Brasil y Uru- guay dan cuenta de DIV conteniendo $300 \mathrm{mg}$ de P4, utilizados hasta en tres oportunidades con porcentajes de celos y ovulación entre $80 \%$ y $100 \%$ en pequeños rumiantes ${ }^{6,8-11}$

Las hembras con DIV de primero y segundo uso tuvieron concentraciones séricas de $\mathrm{P}_{4}$ superiores a los $2 \mathrm{ng} / \mathrm{ml}^{10,11}$. Sin embargo, las concentraciones séricas 
fueron inferiores a $2 \mathrm{ng} / \mathrm{ml}$ en animales con DIV de tercer uso ${ }^{11}$, lo que podría promover el crecimiento excesivo y la persistencia del folículo de mayor tamaño ${ }^{2,12}$.

No existe información sobre las concentraciones de $\mathrm{P}_{4}$ alcanzadas con DIV que contengan menor concentración hormonal inicial ni resultados de estudios farmaco-cinéticos mediante análisis no compartimental de las concentraciones séricas de $\mathrm{P}_{4}$ luego de la colocación de DIV nuevos o usados en ovejas.

El presente trabajo tuvo como objetivo evaluar la utilización y reutilización de DIV con una concentración inicial de $160 \mathrm{mg}$ de $\mathrm{P}_{4}$.

\section{MATERIAL Y MÉTODOS}

\section{Animales}

El estudio se realizó durante la estación reproductiva en el predio de la Facultad de Ciencias Veterinarias de la Universidad Nacional de La Plata, Argentina, de acuerdo con las recomendaciones internacionales especificadas en la guía para el cuidado y uso de los animales de laboratorio ${ }^{3}$ y el Comité Institucional de Cuidado y Uso de Animales de Laboratorio de FCV-UNLP.

Se utilizaron hembras ovinas $3 / 4$ Texel $(n=20)$ con una condición corporal entre 2,5 y 3,0 (1 emaciada a 5 gorda), las cuales fueron alimentadas con heno de alfalfa y balanceado comercial, con acceso ad-libitum al agua de bebida. Se utilizaron DIV nuevos y usados con $300 \mathrm{mg}$ de $\mathrm{P}_{4}$ y con $160 \mathrm{mg} \mathrm{P}_{4}$.

Todos los DIV usados fueron colocados, previo al inicio del experimento, en ovejas durante 7 días para que se consuma el contenido de $\mathrm{P}_{4}$ equivalente a un solo uso. Luego del retiro de los DIV, éstos fueron lavados, sanitarizados y mantenidos en un lugar seco y oscuro hasta su posterior reutilización ${ }^{10}$

Siete días previos al inicio del experimento, todas las hembras recibieron una dosis de $75 \mu \mathrm{g}$ de cloprostenol, el cual se repitió al día 1 con la finalidad de provocar la luteólisis y asegurar la ausencia de $\mathrm{P}_{4}$ endógena durante el experimento. El día 0 correspondió a la colocación del DIV (Figura 2.1). Las ovejas fueron asignadas en forma aleatoria a 5 grupos experimentales: CON: grupo control $(n=4)$ animales sin DIV; CRON: Grupo DIV $160 \mathrm{mg} \mathrm{P}_{4}$ de $1^{\circ}$ uso $(\mathrm{n}=4)$, animales con DIV nuevo; CROU: Grupo DIV $160 \mathrm{mg} \mathrm{P}_{4} 2^{\circ}$ uso $(\mathrm{n}=4)$, animales con DIV usado; CIDN: Grupo DIV $300 \mathrm{mg}$ de $\mathrm{P}_{4}$ de $1^{\circ}$ uso $(\mathrm{n}=4)$, animales con DIV nuevo; CIDU: Grupo DIV $300 \mathrm{mg}$ de $\mathrm{P}_{4}$ de $2^{\circ}$ uso: $(\mathrm{n}=4)$, animales con DIV usado.

Siete días más tarde se retiraron los DIV y al día 18 se aplicó una dosis de $75 \mu \mathrm{g}$ de cloprostenol para inducir la luteólisis y asegurar la ausencia de $\mathrm{P}_{4}$ endógena. En el día 19 se repitió el experimento.

\section{Determinación de progesterona}

En ambas réplicas experimentales, se recolectaron muestras de sangre por venopunción yugular a la hora 0 (antes de la colocación del DIV), a las 12, 24, 36, 48, $72,84,96,108,120,136,144,156$ y 168 h luego del inicio de los tratamientos. Además, se realizaron dos ventanas de sangrado, una primera ventana a las 2, 4, 6,8 y $10 \mathrm{~h}$; y otra a las $170,172,174,176$, y $178 \mathrm{~h}$ del inicio de cada réplica.

Las muestras sanguíneas se centrifugaron durante $10 \mathrm{~min}$ a $2000 \mathrm{rpm}$ y los sueros se almacenaron a $-20^{\circ} \mathrm{C}$ hasta su análisis. Las determinaciones de $\mathrm{P}_{4}$ se realizaron por medio de la técnica de radio-inmuno-ensayo a través de un kit comercial. Los valores de control bajo fueron $0,5,0,7$ y $0,8 \mathrm{ng} / \mathrm{ml}$ y los valores de control alto fueron $5,0,4,5$ y $7,7 \mathrm{ng} / \mathrm{ml}$. El coeficiente de variación (CV) intraensayo fue del 9,4 \% y el CV inter-ensayo fue del $11,6 \%$ para el pool bajo y para el pool alto fue del $4,2 \%$ y del $6,3 \%$, respectivamente.

\section{Análisis estadístico}

Los datos se presentan como cuadrados medios mínimos \pm error estándar. La significancia estadística fue fijada en $\mathrm{P}<0,05$. La oveja fue considerada la unidad experimental. Las concentraciones séricas de $\mathrm{P}_{4}(\mathrm{ng} / \mathrm{ml})$ fueron analizadas mediante análisis de varianza utilizando el procedimiento MIXED de $\mathrm{SAS}^{\circledR}$ como mediciones repetidas en el tiempo ${ }^{8}$. El modelo incluyó el efecto aleatorio de la oveja y el efecto fijo del tiempo (día del tratamiento), tratamiento (CON, CRON, CIDN, CROU, CIDU) y la interacción de tiempo por tratamiento.

El análisis farmaco-cinético de los datos de concentración de $\mathrm{P}_{4}$ en función del tiempo obtenidos, se realizó mediante el programa Phoenix. Los parámetros farmacocinéticos analizados fueron la concentración máxima observada $\left(\mathrm{C}_{\text {max }}\right)$, el tiempo de ocurrencia de la concentración máxima $\left(\mathrm{T}_{\max }\right)$, el área bajo la curva de concentración en función del tiempo desde el día 0 hasta el día 7 ( $\mathrm{ABC}_{0-7 \mathrm{~d}}$ ), el $\mathrm{ABC}$ extrapolado al infinito $\left(\mathrm{ABC}_{0-\infty}\right)$ y el tiempo medio de residencia de la partícula en el sistema (TMR).

El análisis estadístico de los resultados del estudio farmacocinético se realizó mediante análisis de varianza utilizando el procedimiento GLM de $\mathrm{SAS}^{\circledR}$. El modelo incluyó el efecto fijo de tratamiento (CON, CRON, CIDN, CROU, CIDU). El nivel de significancia fue fijado en $\mathrm{P}<0,05$.

\section{RESULTADOS}

Al inicio de cada réplica, las concentraciones séricas de $\mathrm{P}_{4}$ en todos los grupos experimentales fueron menores a $1 \mathrm{ng} / \mathrm{ml}$, confirmando la ausencia de cuerpo lúteo durante el experimento (Figuras 1 y 2). Luego de la inserción de los DIV, el $\mathrm{T}_{\max }$ fue alcanzado entre las 4,5 y $15,7 \mathrm{~h}$, sin presentar diferencias significativas entre grupos (Tabla 1; $>>0,05$ ). La $\mathrm{C}_{\max }$ alcanzada fue similar entre CIDN y CRON (Tabla $1 ; \mathrm{p}>0,05$ ). En cambio, el grupo CIDU tuvo mayor concentración sérica que el grupo CROU (Tabla $1 ; p<0,05$ ).

Así mismo, las hembras del grupo CROU presentaron concentraciones séricas menores a $2 \mathrm{ng} / \mathrm{ml}$ a partir de las $48 \mathrm{~h}$ de colocado el DIV (Figura 2). El TMR fue 


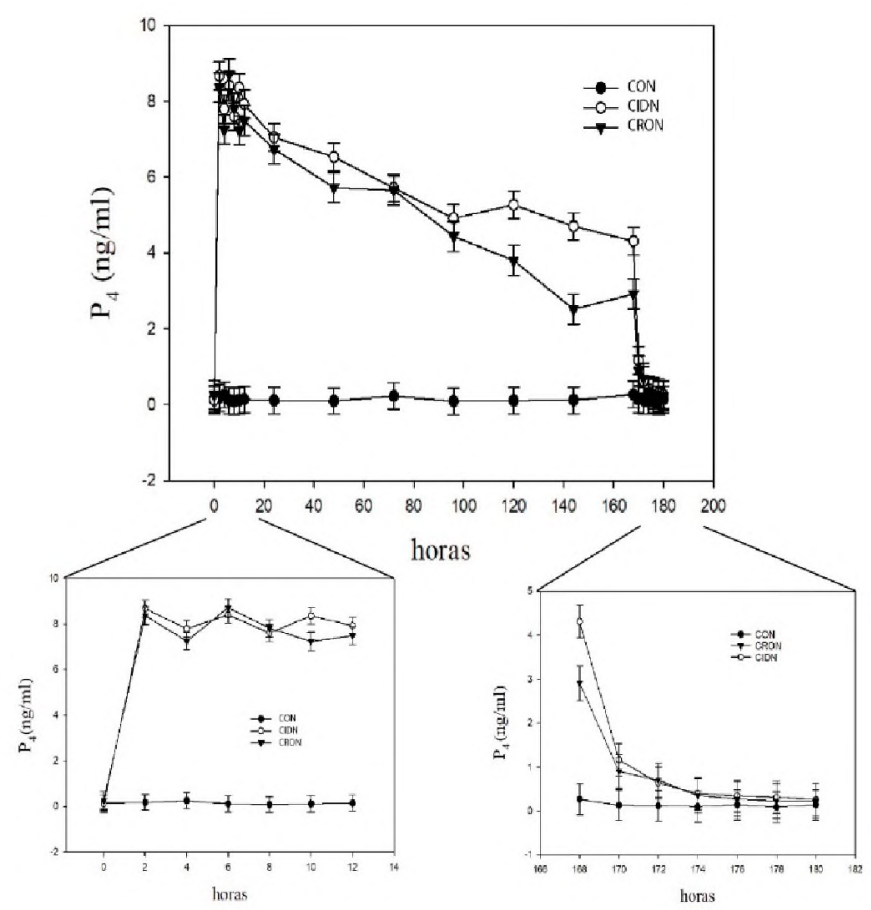

Figura 1. Concentraciones séricas de $\mathrm{P}_{4}(\mathrm{ng} / \mathrm{ml})$ en ovejas luego de la inserción de un DIV nuevo. CON: grupo control; CIDN: DIV $300 \mathrm{mg}$ de $\mathrm{P}_{4}$ de $1^{\circ}$ uso, CRON: DIV $160 \mathrm{mg} \mathrm{P}_{4} 1^{\circ}$ uso.

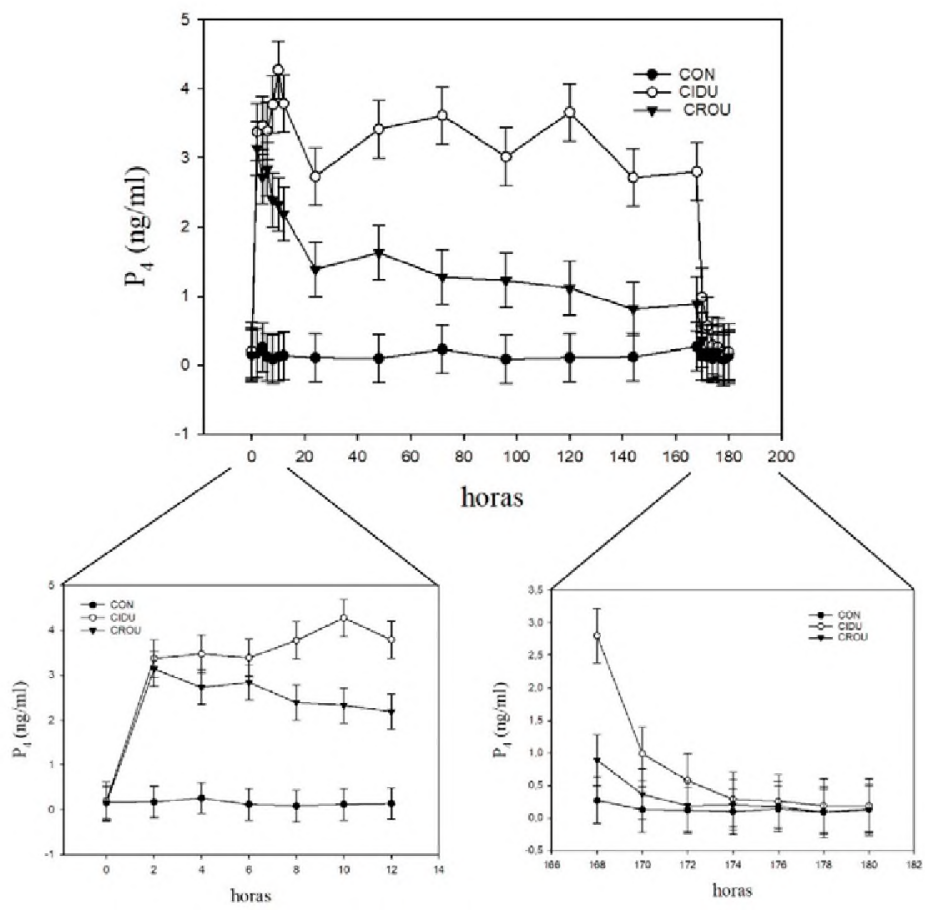

Figura 2. Concentraciones séricas de $\mathrm{P}_{4}(\mathrm{ng} / \mathrm{ml})$ en ovejas luego de la inserción de un DIV usado (cuadrados medios mínimos \pm error estándar). CON: sin DIV; CIDU: DIV $300 \mathrm{mg} \mathrm{P}_{4} 2^{\circ}$ uso y CROU: DIV $160 \mathrm{mg} \mathrm{P}_{4} 2^{\circ}$ uso.

similar entre DIV nuevos y entre DIV nuevos vs. usados (Tabla 1; $p>0,05$ ). En cambio, al comparar entre los DIV usados, el TMR fue mayor en CIDU que en CROU (Tabla 1; $\mathrm{p}<0,05$ ).

El análisis farmacocinético no compartimental determinó que el $\mathrm{ABC}_{0 \text {-7d }}$ fue similar entre grupos CIDN y CRON (p: 0,05; Tabla 1). Asímismo, los grupos CIDU y CROU presentaron diferencias significativas entre sí $(\mathbf{p}<0,05)$ y con los grupos con DIV nuevos $(p<0,05$; Ta- bla 1).En términos absolutos, el CIDU liberó un $41,20 \%$ menos de $\mathrm{P}_{4}$ que el CIDN (Figura 2.3), y el CROU liberó un 72,5\% menos de $\mathrm{P}_{4}$ que CRON (Figura 2.3).

A pesar de que no hubo diferencias significativas en la liberación de $\mathrm{P}_{4}$ entre los CIDN y CRON, el CROU liberó un 68,5\% menos de $\mathrm{P}_{4}$ que el CIDU (Figura 2.3) $\mathrm{Al}$ analizarse el $\mathrm{ABC}_{0-\infty}$, se tuvieron resultados similares a los correspondientes al $\mathrm{ABC}_{0-7 \mathrm{~d}^{\circ}}$ 
Tabla 1 Parámetros farmacocinéticos de las concentraciones séricas de $\mathrm{P}_{4}$ tras la aplicación de DIV nuevo y usado (cuadrado medio mínimo \pm error estándar) en ovejas.

\begin{tabular}{lccccc}
\hline grupo & $\begin{array}{c}\mathrm{ABC}_{0-7} \\
(\mathrm{ng} \mathrm{h} / \mathrm{ml})\end{array}$ & $\begin{array}{c}\mathrm{ABC}_{0-\infty} \\
(\mathrm{ng} \mathrm{h} / \mathrm{ml})\end{array}$ & $\begin{array}{c}\mathrm{C}_{\max } \\
(\mathrm{ng} / \mathrm{ml})\end{array}$ & $\begin{array}{c}\mathrm{T}_{\max } \\
(\mathrm{h})\end{array}$ & $\begin{array}{c}\text { TMR } \\
(\mathrm{h})\end{array}$ \\
\hline CIDN & $980,73 \pm 88,19^{\mathrm{a}}$ & $982,37 \pm 88,53^{\mathrm{a}}$ & $10,80 \pm 1,03^{\mathrm{a}}$ & $7,00 \pm 1,06$ & $75,64 \pm 2,49$ \\
CRON & $822,93 \pm 103,54^{\mathrm{a}}$ & $830,56 \pm 103,12^{\mathrm{a}}$ & $10,18 \pm 1^{\mathrm{a}}$ & $4,50 \pm 0,82$ & $68,85 \pm 2,24$ \\
CIDU & $586,48 \pm 66,39^{\mathrm{b}, \mathrm{c}}$ & $589,42 \pm 65,63^{\mathrm{b}, \mathrm{c}}$ & $5,51 \pm 0,86^{\mathrm{b}, \mathrm{c}}$ & $15,75 \pm 8,16$ & $81,22 \pm 1,55^{\mathrm{c}}$ \\
CROU & $226,15 \pm 33,16^{\mathrm{b}, \mathrm{d}}$ & $231,00 \pm 36,12^{\mathrm{b}, \mathrm{d}}$ & $3,48 \pm 0,40^{\mathrm{b}, \mathrm{d}}$ & $6,25 \pm, 571$ & $71,04 \pm 1,74^{\mathrm{d}}$ \\
\hline
\end{tabular}

$\mathrm{ABC}_{0-7}$ : área bajo la curva concentración hasta $\operatorname{los} 7 \mathrm{~d}$. $\mathrm{ABC}_{0-\infty}$ : área bajo la curva concentración extrapolada al infinito. $\mathrm{C}_{\max }$ : concentración sérica máxima. $\mathrm{T}_{\max }$ : tiempo a la concentración máxima. TMR: tiempo medio de residencia. Valores en una columna con diferentes superíndices, difieren al $\mathrm{p}<0.05$.

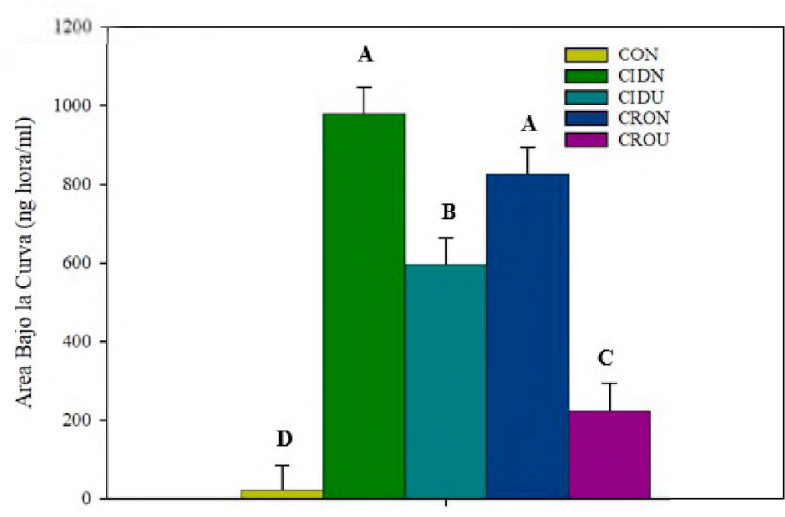

Figura 3. Área bajo la curva de las concentraciones séricas de $\mathrm{P}_{4}$ (ng hora/ml) en ovejas.CON: sin DIV, CRON: DIV $160 \mathrm{mg} \mathrm{P}_{4} 1^{\circ}$ uso, CIDN, DIV $300 \mathrm{mg}$ $\mathrm{P}_{4} 1^{\circ}$ uso, CROU: DIV $160 \mathrm{mg} \mathrm{P}_{4} 2^{\circ}$ uso, CIDU: DIV $300 \mathrm{mg} \mathrm{P}_{4} 2^{\circ}$ uso. Barras con diferentes letras difieren al $\mathbf{p}<0.05$.

\section{DISCUSIÓN}

En nuestro conocimiento, este es el primer trabajo que realiza un estudio sobre la farmacocinética mediante análisis no compartimental de las concentraciones séricas de $\mathrm{P}_{4}$ en DIV nuevos o usados. De los DIV con $160 \mathrm{mg} \mathrm{P}_{4}$ utilizados, sólo las ovejas del grupo CRON presentaron concentraciones de $\mathrm{P}_{4}>2 \mathrm{ng} / \mathrm{ml} \mathrm{du}-$ rante el período de 7 días estudiados mientras que el grupo CROU presentó concentraciones sub-luteales de $\mathrm{P}_{4}(<2 \mathrm{ng} / \mathrm{ml})$ a partir de las $48 \mathrm{~h}$ de colocado el DIV.

Estas concentraciones séricas hormonales, posiblemente promoviesen el crecimiento excesivo y la persistencia del folículo de mayor tamaño, así como el envejecimiento del folículo ovulatorio ${ }^{3,12}$. Si bien algunos trabajos previos estudiaron los perfiles séricos de $\mathrm{P}_{4}$ con DIV $300 \mathrm{mg}$ de $\mathrm{P}_{4}$ nuevos y usados, estos estudios no incluyeron los estudios farmacocinéticos mediante análisis no compartimental del $\mathrm{ABC}_{0-7}, \mathrm{el}^{\mathrm{ABC}_{0-\infty}}, \mathrm{C}_{\max }$ alcanzada, y el TMP de $\mathrm{P}_{4}$.

Los resultados obtenidos a partir de las concentraciones séricas de $\mathrm{P}_{4}$ de los grupos CIDN y CIDU son coincidentes con trabajos previos ${ }^{1,5,11}$. Mientras que en éste y otros estudios previos las concentraciones sé- ricas de $\mathrm{P}_{4}$ alcanzadas con CIDN en ovejas fueron similares ${ }^{12}$, en nuestro estudio las concentraciones alcanzadas por CIDU fueron más bajas ${ }^{12}$. Los estudios de farmacocinética no compartimental realizados en este trabajo no están disponibles para las labores previas y por lo tanto no se puede realizar una comparación.

En base a los resultados de este trabajo, la reutilización de DIV con $160 \mathrm{mg}$ de $\mathrm{P}_{4}$ no sería efectiva ya que las concentraciones séricas de $\mathrm{P}_{4}$ serían un tercio de las producidas en hembras con un DIV nuevo y con valores $<2 \mathrm{ng} / \mathrm{ml}$ durante un periodo de tratamiento de 7 días, lo que afectaría la dinámica y el crecimiento folicular, causando un excesivo desarrollo y persistencia en el tiempo de los folículos y posiblemente disminuyendo la fertilidad.

\section{REFERENCIAS}

1. Ainsworth L, Downey BR. 1986. A controlled internal drug-release dispenser containing progesterone for control of the estrous cycle of ewes. Theriogenology 26: 6, 847-856

2. Johnson SK, Dailey RA, Inskeep EK, Lewis PE. 1996. Effect of peripheral concentrations of progesterone on follicular growth and fertility in ewes. Domestic Animal Endocrinology 13: 1, 69-79.

3. NRC. 1986. Guide for the care and use of laboratory animals. US Department of Health and Human Services, Public Health Service, $8^{\circ}$ ed., Washington, 2011.

4. Oliveira MA, Guido SI, Lima PF. 2001. Comparison of different protocols used to induce and synchronize estrus cycle of Saanen goats. Small Ruminant Research 40: 2, 149-153.

5. Pinna AE et al. 2012. Reproductive parameters of Santa Inês ewes submitted to short-term treatment with re-used progesterone devices. Arqu Bras Med Vet e Zoot 64: 2, 333-340.

6. Rathbone MJ, Macmillan KL, Bunt CR, Burggraaf S. 1997. Conceptual and commercially available in travaginal veterinary drug delivery systems. Adv Drug Deliv Reviews 28: 3, 363-392.

7. SAS Institute. 2015. Base SAS 9.4 procedures guide. SAS Institute, Cary, North Carolina, United States. 
8. Uribe LF et al. 2011. Reutilización del dispositivo de progesterona (CIDR) asociado con protocolos de corta duración en cabras. Vet Zootec 5: 1, 39-46.

9. Vilariño M, Rubianes E, Lier E, Menchaca A. 2010. Serum progesterone concentrations, follicular development and time of ovulation using a new progesterone releasing device (DICOß) in sheep. Small Rum Res 91: 2-3, 219-224.

10. Vilariño M, Rubianes E, Menchaca A. 2011. Re-use of intravaginal progesterone devices associated with the short-term protocol for timed artificial insemination in goats. Theriogenology 75: 7, 1195-1200.
11. Vilariño M, Rubianes E, Menchaca A. 2013. Ovarian responses and pregnancy rate with previously used intravaginal progesterone releasing devices for fixed-time artificial insemination in sheep. Theriogenology 79: 1, 206-210

12. Viñoles C, Meikle A, Forsberg M, Rubianes E. 1999 The effect of subluteal levels of exogenous progesterone on follicular dynamics and endocrine patterns during the early luteal phase of the ewe. Theriogenology 51: 7, 13511361.

\section{Revista Veterinaria ingresa a SciELO}

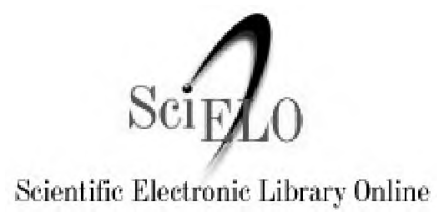

Revista Veterinaria, publicación oficial de la Facultad de Ciencias Veterinarias de la Universidad Nacional del Nordeste (Corrientes, Argentina), ha logrado acceder al Núcleo Básico de Revistas Científicas Argentinas (Nivel 1), luego de calificar adecuadamente en el Centro Argentino de Información Científica y Tecnológica (CAICYT), según Resolución 2485/14 del Consejo Nacional de Investigaciones Científicas y Técnicas (CONICET).

Sobre un puntaje máximo de 33 se obtuvieron 32 puntos. Tal calificación constituye "una garantía de la excelencia de la publicación" (sic) y queda expedita la vía del Portal SciELO (Scientific Electronic Library Online) para los artículos publicados.

En tal calificación gravitó positivamente la circunstancia de haber aumentado el índice de impacto (Scimago-Elsevier) y haber disminuido las autocitaciones. También se tuvieron en cuenta aspectos como la amplia cobertura de la revista, la calidad científica del Comité Editorial, los criterios de evaluación de los artículos, el origen de los autores (locales $60 \%$, nacionales $13 \%$, extranjeros $24 \%$, en idioma inglés), el adecuado balance entre trabajos científicos originales y reseñas bibliográficas (ambos con alta calidad), así como el estricto cumplimiento de la periodicidad semestral y la favorable acogida por indizadores como Cab, J-Gate, Doaj, Ebsco, Gale Cengage, Infocyt, Latindex y Scopus.

Se consolida de esta manera la continuidad de "Revista Veterinaria", que en su acontecer registra más de 50 años de existencia en nuestra Facultad de Ciencias Veterinarias, entidad que en 2019 cumplió el $99^{\circ}$ aniversario de su fundación. 